\title{
Complex Nature of Enterococcal Pheromone-Responsive Plasmids
}

\author{
EWA WARDAL ${ }^{* 1}$, EWA SADOWY $^{2}$ and WALERIA HRYNIEWICZ ${ }^{1}$ \\ ${ }^{1}$ Department of Epidemiology and Clinical Microbiology \\ ${ }^{2}$ Department of Molecular Microbiology, National Medicines Institute, Warsaw, Poland
}

Received 11 March 2010, accepted 15 April 2010

\begin{abstract}
Pheromone-responsive plasmids constitute a unique group of $\sim 20$ plasmids identified, as yet, only among enterococcal species. Several of their representatives, e.g. pAD1, pCF10, pPD1 and pAM373 have been extensively studied. These plasmids posses a sophisticated conjugation mechanism based on response to sex pheromones - small peptides produced by plasmid-free recipient cells. Detailed analysis of regulation and function of the pheromone response process revealed its great complexity and dual role - in plasmid conjugation and modulation of enterococcal virulence. Among other functional modules identified in pheromone plasmids, the stabilization/partition systems play a crucial role in stable maintenance of the plasmid molecule in host bacteria. Among them, the par locus of pAD1 is one of the exceptional RNA addiction systems. Pheromone-responsive plasmids contribute also to enterococcal phenotype being an important vehicle of antibiotic resistance in this genus. Both types of acquired vancomycin resistance determinants, vanA and vanB, as well many other resistant phenotypes, were found to be located on these plasmids. They also encode two basic agents of enterococcal virulence, i.e. aggregation substance (AS) and cytolysin. AS participates in mating-pair formation during conjugation but can also facilitate the adherence of enterococci to human tissues during infection. The second protein, cytolysin, displays hemolytic activity and helps to invade eukaryotic cells. There are still many aspects of the nature of pheromone plasmids that remain unclear and more detailed studies are needed to understand their uniqueness and complexity.
\end{abstract}

K e y word s: pheromone-responsive plasmid, sex pheromone, inhibitory peptide, aggregation substance, vancomycin resistance

\section{Introduction}

Enterococci are Gram-positive bacteria, inhabiting gastrointestinal tracts of humans and animals. They are also commonly found in food, soil, sewage and water. During the last three decades enterococci have emerged as opportunistic, multi-resistant pathogens, causing hospital infections, especially in immunocompromised patients with two most prevalent species, Enterococcus faecalis and Enterococcus faecium (CDC, 2004). Treatment of enterococcal infections is frequently complicated by their resistance to antimicrobials of several classes (Murray, 1998). Acquired resistance in enterococci is often associated with mobile genetic elements (MGE), especially plasmids, i.e. autonomously replicating, extrachromosomal DNA molecules, able to be stably maintained in consecutive bacterial generations and to disseminate in the bacterial population.

A wide spectrum of replicons can be distinguished among enterococcal plasmids, ranging from small cryptic plasmids to megaplasmids, composed of differ- ent functional modules, responsible for plasmid replication, maintenance, conjugal transfer and phenotypic traits such as resistance to antimicrobials. Three plasmid types are known to replicate in enterococci: broad host range RCR (rolling circle replicating) and Inc 18 plasmids, and limited to enterococci pheromoneresponsive plasmids. This review will focus on the family of $\sim 20$ pheromone-responsive plasmids, which display exceptional features, typical only for this group of elements. Pheromone-responsive plasmids described thus far range in size from 37 to $128 \mathrm{~kb}$, have a low copy number (2-4 per cell) and evolved a specific conjugation mechanism based on pheromones, restricted to enterococci (Weaver et al., 2002).

\section{Conjugation system - basic features and regulation mechanism}

One of the most extensively studied properties of pheromone-responsive plasmids is their unique conjugation mechanism. The principle of every conjugation

* Corresponding author: E. Wardal, Department of Epidemiology and Clinical Microbiology, National Medicines Institute, Chełmska 30/34, 00-725 Warsaw, Poland; phone/fax: (+48) 228514388 / (+48) 2284106 52; e-mail: ejagiello@cls.edu.pl 
mechanism is the formation of couples of bacterial cells, which facilitates the transfer of plasmid DNA from donor to recipient cell through a special multiprotein complex formed in the region of cell contact. Conjugation functions are encoded by the tra region of a plasmid, which in Gram-positive species exhibits homology to genes encoding type IV secretion systems. Several components encoded by this region are responsible for plasmid DNA binding, ATP-dependent translocation of single-stranded DNA or the formation of mating channel (Grohmann et al., 2003).

Enterococcal pheromone-responsive plasmids constitute one of the most original bacterial MGE. The conjugation mechanism of these plasmids, discovered over 30 years ago (Dunny et al., 1978) is based on the existence of special molecules called sex pheromones - small extracellular peptides specific for donors carrying various conjugative plasmids. Sex pheromones are produced by potential recipients, i.e. bacterial cells that lack a plasmid from a particular pheromone group. These signal molecules are chromosomally encoded, whereas genetic determinants for signal detection and response reside on the sex pheromone plasmid (Kozlowicz et al., 2006). Donor cell in the presence of the pheromone produces proteinaceous structures on the cell surface (Yagi et al., 1983) called aggregation substance (AS) which binds to enterococcal binding substance (EBS) present on the surface of the recipient. During this process, a mating channel between the donor and the recipient is formed, which enables transfer of the plasmid DNA. After acquiring the plasmid, the recipient cell shuts down the production of pheromone and begins to produce a specific, plasmid-encoded inhibitor peptide which serves to de- sensitize the bacterial cell to low levels of endogenous pheromone and exogenous pheromones produced by donors (Mori et al., 1986). Special surface exclusion proteins are exposed on the bacterial cells that prevent from acquiring the plasmids already present in the cell (entry-exclusion mechanism). Their probable role is blocking plasmid DNA transfer by disturbing matingpair formation between donors (Clewell, 1993).

The pheromone-responsive conjugation system has been most extensively examined for plasmids pAD1, pCF10, pPD1 and pAM373 from E. faecalis. A schematic representation of conjugation steps of these plasmids and key molecular determinants of each system are listed in Table I. One of the first pheromoneresponsive plasmids discovered was highly conjugative 60-kb plasmid pAD1, harboring several resistance and virulence determinants (Clewell, 2007), identified in E. faecalis DS16 clinical isolate. More than a half of pAD1 is devoted to mating response and regulation of this process (Francia and Clewell, 2002) and exhibits high level of homology to equivalent regions of other sex pheromone plasmids (Hirt et al., 1996). All major steps, leading to the acquisition of pAD1 by plasmidfree cells, are very similar in most pheromone responsive plasmids characterized so far. Interestingly, plasmid pAM373 exhibits discrepancies in gene content and functions. The AS coded on this plasmid is structurally different and is able to bind to cells defective in EBS (Muscholl-Silberhorn, 1999). Another feature of pAM373 is the absence of the entry-exclusion function (de Boever and Clewell, 2001).

The first evidence for the existence of special molecules inducing cell aggregation and mating has been shown by Dunny et al. (1978). Working on E. faecalis,

Table I

Schematic representation of conjugation steps of four best-characterized pheromone-responsive plasmids with specification of key molecular determinants of conjugative functions.

\begin{tabular}{|c|c|c|c|c|}
\hline \multirow[t]{2}{*}{ Description of conjugation step } & \multicolumn{4}{|c|}{$\begin{array}{l}\text { Nomenclature of proteins and relevant genes involved } \\
\text { in conjugation steps in different plasmids }\end{array}$} \\
\hline & pAD1 & pCF10 & pPD1 & pAM373 \\
\hline 1. Secretion of chromosomally-encoded sex pheromones & cAD1 $(c a d)$ & $\mathrm{cCF} 10(\operatorname{ccf} A)$ & cPD1 $(c p d)$ & cAM373 (camE) \\
\hline $\begin{array}{l}\text { 2. Recognition and internalization of pheromones } \\
\text { by recipient cell surface }\end{array}$ & $\operatorname{TraC}$ & $\operatorname{PrgZ}$ & TraC & TraC \\
\hline 3. Induction of conjugation system & TraA & $\operatorname{PrgX}$ & TraA & TraA \\
\hline $\begin{array}{l}\text { 4a. Synthesis of aggregation substance (AS) } \\
\text { on the donor cell }\end{array}$ & Asal (asal) & $\operatorname{Asc10}(\operatorname{prg} B)$ & Asp (asp) & Asa373 (asa373) \\
\hline $\begin{array}{l}\text { 4b. Aggregation of donors and recipients - binding of AS } \\
\text { and BS (binding substance on recipient cell) }\end{array}$ & Sea1 (seal) & $\operatorname{PrgA}(\operatorname{Sec} 10)$ & Sep1 (sep1) & none \\
\hline 5. Initiation of plasmid transfer & $\begin{array}{l}\text { oriT1, oriT2, } \\
\text { TraX, TraW }\end{array}$ & oriT, PcfG & $n k$ & $\begin{array}{l}\text { oriT, TraX and } \\
\text { TraW homologs }\end{array}$ \\
\hline $\begin{array}{l}\text { 6. Shut down of conjugation functions } \\
\text { in plasmid-acquired recipient }\end{array}$ & iAD1 (iadl) & iCF10 $(\operatorname{prg} Q)$ & iPD1 (ipd) & iAM373 (iam373) \\
\hline
\end{tabular}

Proteins assigned to subsequent steps are as follows: 1 - sex pheromones, 2 - bacterial surface receptors, 3 - negative regulator of conjugation system, $4 \mathrm{a}$ - aggregation substance, $4 \mathrm{~b}$ - binding substance, 5 - origins of transfer, relaxases and TraG-like proteins, 6 - inhibitory peptides (Clewell and Dunny, 2002; Ozawa et al., 2005; Chandler et al., 2005; Folli et al., 2008; Clewell, 2007); nk, not known. 


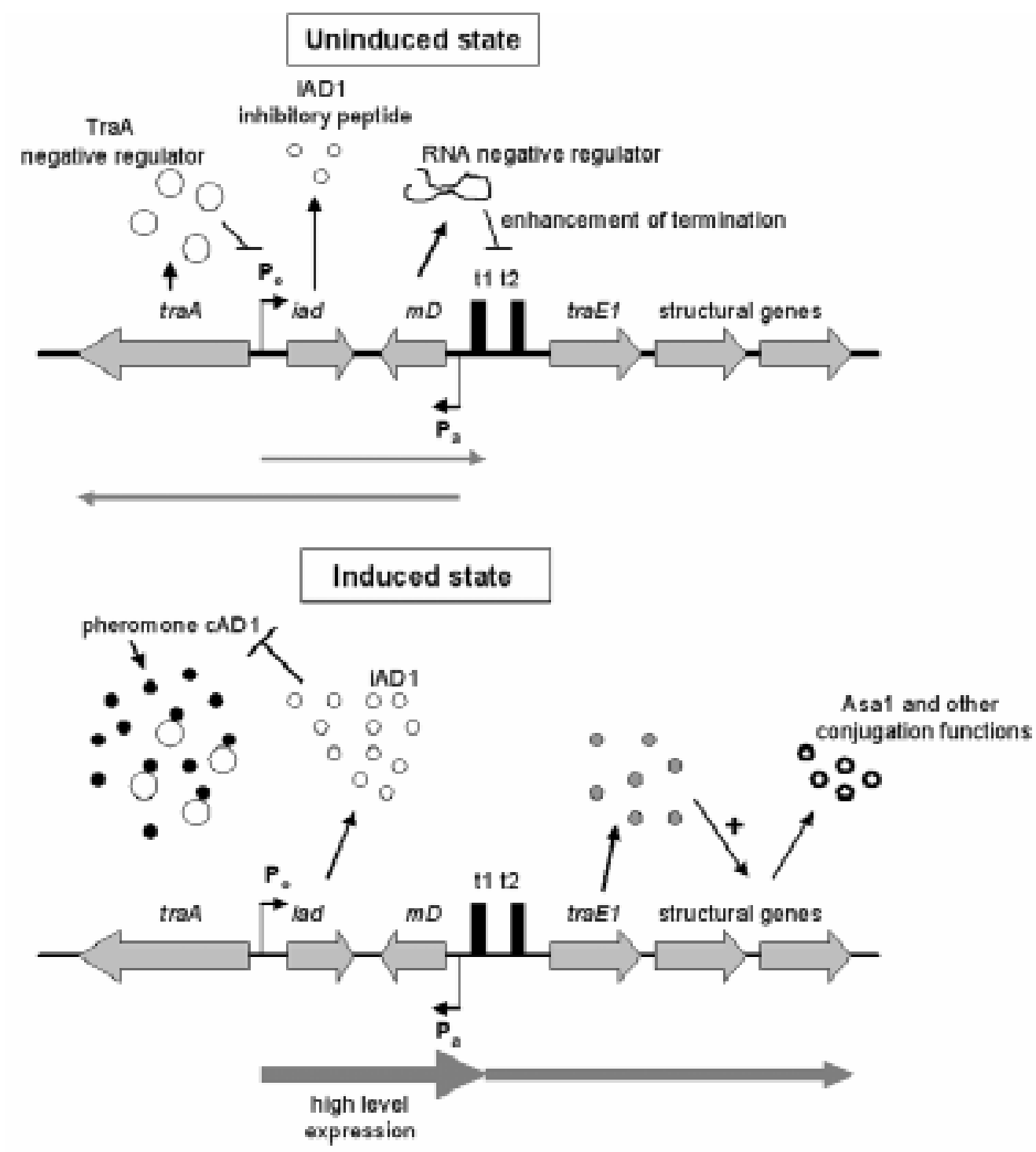

Fig.1. Schematic representation of regulation mechanism of pAD1 pheromone response.

In the absence of pheromone two negative regulators - TraA and $\mathrm{mD}$ keep the expression of iad on a very low level and block the transcription of TraE1 and structural genes (a). When the pheromone appears it bounds the TraA and negative regulation is abolished, which leads to high level of $\mathrm{iAD} 1$ synthesis and expression of TraE1 and conjugation machinery proteins (b). TraA, $\mathrm{mD}$ - negative regulators, TraE1 - positive regulator, iad - iAD1 gene, t1, t2 - termination sites, $\mathrm{P}_{\mathrm{o}}, \mathrm{P}_{\mathrm{a}}$ - opposite promoters (according to Clewell and Dunny, 2002; Clewell 2007).

they discovered heat-stable, protease sensitive factors that were called sex pheromones. Different pheromones produced by strains lacking the particular plasmid, exhibit high specificity to the corresponding pheromone-responsive plasmids. One bacterial cell may secrete at least six different pheromones (Clewell and Dunny, 2002). This production results in conjugation and plasmid acquisition, which stops the secretion of a particular pheromone. However, synthesis of other pheromones is continued and thus single bacterial cell is able to accumulate different pheromoneresponsive plasmids (Wirth, 1994). Pheromone plasmids can be divided into subgroups on the basis of their pheromone sensitivity, which parallels plasmid incompatibility characteristics (Hirt et al., 1996).

Although the structure of sex pheromone molecules was described many years ago, all that was known about their precursors came down to the statement that they must be chromosomally-encoded, since they are excreted by plasmid-free cells (Wirth, 1994). Finally, Clewell et al. (2000) identified pheromone determinants. It was revealed that pheromones are part of signal sequences of lipoprotein precursors. In the case of cAD1 (coded by the cad determinant), the mature peptide corresponds to the last 8 residues of the 22-amino acid signal sequence of the 309 aa lipoprotein precursor (An and Clewell, 2002). The generation of pheromone molecule involves the action of lipoprotein signal peptidase and the Eep protein similar to certain metaloproteases (Brown et al., 2000). The identification of pheromone inhibitory peptide precursors, coded on plasmids, took place very early, e.g. for pAD1, the inhibitor iAD1 is coded by the iad determinant. The mature iAD1 peptide corresponds to the last 8 residues of the 22 aa 
precursor (Clewell et al., 1990). Inhibitor and corresponding pheromone have the same length and similar sequences, LFSLVLAG and LFVVTLVG, respectively.

The majority of other known pheromones and pheromone inhibitors display great similarity in their processing mechanism and final structure (Clewell et al., 2000), except for cAM373, which is produced independently of Eep activity (An et al., 1999) and represents the only known example when a very similar peptide is excreted by bacteria other than enterococci, namely by staphylococci (Clewell et al., 1985). Nevertheless, there is no relationship between lipoprotein precursors of cAM373 and staph-cAM373 (camE and camS, respectively), suggesting the similarity between these two peptides is only coincidental (Flannagan and Clewell, 2002).

Potential recipient cells produce constitutively high amounts of pheromone molecules. However, low level of endogenously produced pheromones is observed also for plasmid donors. Strict and complex regulation of conjugation functions prevents self-induction of pheromone-responsive plasmids. As yet, these regulatory mechanisms have been extensively studied for four plasmids - pAD1, pCF10, pPD1 and pAM373 (Clewell and Dunny, 2002). The key players of each system are: (i) two essential intracellular negative regulators, which control the gene expression from two opposite promoters and (ii) positive regulator, controlling the synthesis of conjugation structural genes. In the absence of inducing level of pheromone (Fig. 1a), a negative regulatory protein TraA blocks $\mathrm{P}_{\mathrm{o}}$ - the promoter for the iad gene (iAD1 determinant). As a result, inhibitory peptide is expressed at a very low level allowing for titration of endogenous pheromone in the uninduced state (Pontius and Clewell, 1992). The transcription from $P$ goes until $\mathrm{t} 1$ and $\mathrm{t} 2$ termination sites but not further (Tomita and Clewell, 2000), due to the presence of the second negative regulator, antisense $\mathrm{RNA} \mathrm{mD}$ expressed at a relatively high level from the convergent promoter $\mathrm{P}_{\mathrm{a}}$. The expression from this promoter provides also a low level of TraA, essential for $\mathrm{P}_{\mathrm{o}}$ activity inhibition (Clewell and Dunny, 2002). $\mathrm{mD}$ is the type of transcription termination enhancer at $\mathrm{t} 1$, because of its $11 \mathrm{nt}$ sequence complementary to a region spanning the t1 "hairpin loop" transcript (Tomita and Clewell, 2000). This complex negative regulatory circuit, based on the transcription and counter-transcription from two opposing promoters $\mathrm{P}_{0}$ and $\mathrm{P}_{\mathrm{a}}$, seems to be a significant feature of the regulation of pheromone response in general (Callen et al., 2004).

When a higher amount of exogenous pheromone appears (Fig. 1b), it is bound by donor cell surface and transported to the cytoplasm, where it binds TraA, switching off its negative regulatory function by release of the protein from the $\mathrm{P}_{\mathrm{o}}$ promoter (Fujimoto and Clewell, 1998).The resulting higher level of transcription from $\mathrm{P}_{\mathrm{o}}$ leads to the elevated production of inhibitory peptide iAD1, as well as transcriptional read-through of $t 1$ and $t 2$ and the expression of positive regulator TraE1, followed by the expression of conjugation structural genes e.g. asal, encoding the AS. As a result of the above-mentioned events, a recipient cell forms aggregates with the donor and acquires the plasmid. The presence of high amount of iAD1 results in a fast shut down of conjugation functions. This sophisticated control mechanism enables response to a slight change in the balance of the pheromone and inhibitory peptides, ensuring high level of sensitivity towards the presence of recipient cells (Kozlowicz et al., 2006).

\section{Other functional modules of pheromone-responsive plasmids}

The conjugation system enables efficient plasmid dissemination in a bacterial population. However, there are several other functional modules present on plasmids, essential for their stable maintenance in a bacterial cell including replication, partition and stabilization systems.

Four most studied pheromone plasmids, namely pCF10, pAD1, pAM373 and pPD1, serve as objects for analysis of the replication machinery. Key determinants of pheromone-responsive plasmids are repA, rep $B$ and repC. RepA plays a role as a replication initiator protein and resembles a family of proteins encoded by several low-copy plasmids from Grampositive bacteria which exhibit a theta-type replication (Francia et al., 2004). The RepB and RepC proteins represent a partition system (Weaver et al., 2002).

pAD1, as well as other pheromone plasmids (e.g. pCF10, pAM373, pPD1, pTEF1, pTEF2) belong to RepA_N family of replicons, known to be broadly distributed in other than enterococci low G+C Gram-positive bacteria, such as staphylococci, lactococci, streptococci and others, as well as in a few phages (Weaver et al., 2009). This group exhibits a narrow host range, which indicates that its evolution took place in native hosts with limited number of genetic exchange between different elements belonging to this family. Genes encoding initiator proteins bear a centrally located region of repeats, called oriV, which serves as the origin of replication. The oriV region of different pheromone responsive plasmids shows sequence variability within the repeats. pCF10 and pPD1 are an interesting example of compatible plasmids with oriV sequences differing only by a single nucleotide, which suggests the role of oriV as incompatibility determinant and provides a good explanation of coexistence of several related pheromone plasmids in a single cell. 
The rep $B$ and rep $C$ determinants, located adjacent to the rep $A$ gene, are required for maximal replicon stabilization (Francia et al., 2007). The series of short repeats, called iterons, flank the rep $B C$ coding region. Their sequence and arrangement vary in different plasmids (Weaver et al., 2002). The basic mechanism of RepBC action is the formation of RepC-iteron complex followed by binding of RepB ATPase, which presumably facilitates the connection between plasmid partition complex and host segregational apparatus as suggested for other partition systems (Funnell and Slavcev, 2004).

Another mechanism, called addiction or toxinantitoxin system (TAS), encoded by number of plasmids prevents plasmid loss from the bacterial population. Two basic types of TAS can be distinguished depending on the type of their components. So called "proteic" systems consist of stable toxin and labile antitoxin. The second type is RNA - regulated addiction system, in which toxin is a protein while antitoxin is present in the form of the RNA molecule transcribed from the antisense strand. The translation of toxin mRNA is blocked in the presence of homologous antitoxin RNA by formation of duplex, subsequently degraded by bacterial RNAse. Bacterial cell lacking plasmid is killed by toxin translated in the absence of antitoxin RNA (Zielenkiewicz and Ceglowski, 2001). Among TAS of pheromone-responsive plasmids, the par locus in pAD1 is best characterized. It represents the unusual type of RNA regulated addiction system in which the binding of two RNAs leads rather to the formation of a highly stable complex than to RNA degradation (Weaver et al., 2004). Regulation of the par locus is based on the small regions of homology between two transcripts: RNAI (transcript for the Fst toxin) and RNAII (small antisense RNA). They are transcribed convergently and overlap at 3 ' end at bidirectional transcription terminators and at 5 ' ends, where similar direct repeats are present. These regions of homology result in the formation of stable RNAI-RNAII complex and inhibition of the Fst expression. Translationally inhibited RNAI is accumulated in the cell. When plasmid replicon is lost, less stable RNAII is degraded and the Fst protein expression leads to membrane permeabilization and cessation of macromolecular synthesis, causing death of the bacterial cell (Weaver et al., 2003).

\section{Dissemination of antimicrobial resistance}

One of the most threatening features of enterococci is their resistance to multiple antibiotics, which makes enterococcal infections difficult to treat. Among resistant enterococcal strains, VRE (vancomycin-resistant enterococci) are considered alert hospital patho- gens (Courvalin, 2005). VRE exhibit high-level resistance to glycopeptides such as vancomycin and teicoplanin, conferred by vanA and vanB determinants (Courvalin, 2006) usually carried on transposons that can integrate into plasmids.

The most commonly detected vancomycin resistance determinants associated with pheromone-responsive plasmids are the vanA operons. E. faecium is the species, in which the first vancomycin resistant pheromone responsive plasmid pHKK100 was reported (Handwerger et al., 1990). An interesting situation was described in another E. faecium strain R7 carrying two plasmids, pHKK702 $(41 \mathrm{~kb})$ with integrated Tn1546 transposon and pheromone-responsive pHKK703 (55 kb; Heaton et al., 1996). When R7 strain was used as a donor in mating experiments, a highly conjugative vancomycin-resistant transconjugant was isolated, which harbored 92-kb plasmid pHKK701, a cointegrate of pHKK702 and pHKK703. Yet another VanA pheromone-responsive plasmid, pBRG1 from the E. faecium LS10 strain was shown to be transferable to E. faecalis and responsive to CCF10 (Magi et al. 2003).

VanA pheromone-responsive plasmids have been described also for E. faecalis. Good examples are two plasmids - pSL1 and pSL2 - isolated in Korea from a patient and chicken feces, respectively (Lim et al., 2006). These plasmids transferred resistance not only to vancomycin but also to gentamicin, kanamycin, streptomycin and erythromycin to E. faecalis strains at high frequency. Both plasmids, $128,1 \mathrm{~kb}$ in size, exhibited identical restriction patterns while their hosts were unrelated which highlights the possibility of conjugative transfer of vancomycin resistance between chicken and human enterococci. Another E. faecalis VanA plasmid, pAM368 $(107 \mathrm{~kb})$ conferred a pheromone response to synthetic cAM373, and culture filtrates of E. faecalis and S. aureus (Showsh et al., 2001), providing the first example of enterococcal resistance determinant located on the plasmid that responds to $S$. aureus cAM373-like peptide and suggested that transfer of this resistance from enterococci to staphylococci cannot be neglected.

Very recently, the VanB type of glycopeptide resistance was described on pheromone-responsive plasmids. In two E. faecalis NKH15 strains, plasmids pMG2200 (106 kb) and pMG2201 (65,7 kb) were detected (Zheng et al., 2009). pMG2200 containing a Tn1549-like element responded to cCF10 and harbored the chimeric conjugation regulatory region, consisting of genes from pCF10 and pAD1.

Not only vancomycin resistance disseminates by pheromone plasmid vehicles. Several examples of pheromone plasmids with resistance determinants are presented in Table II. One of the first pheromoneresponsive plasmids characterized, pCF10, encodes the resistance to tetracycline on Tn925 (Christie and 
Table II

Representatives of pheromone-responsive plasmids and their resistance determinants.

\begin{tabular}{|c|c|c|c|c|}
\hline Plasmid & Original host & Size $(k b)$ & Resistances & References \\
\hline pCF10 & E. faecalis SF-7 & 65 & $\mathrm{Tc}^{\mathrm{R}}$ & Christie and Dunny, 1986 \\
\hline pBEM10 & E. faecalis $\mathrm{HH} 2$ & 70 & $\mathrm{bla}^{+} \mathrm{Cm}^{\mathrm{R}} \mathrm{Km}^{\mathrm{R}} \mathrm{Tm}^{\mathrm{R}}$ & Murray et al., 1988 \\
\hline pAM323 & E. faecalis $\mathrm{HH} 2$ & 66 & $\mathrm{Em}^{\mathrm{R}}$ & Murray et al., 1988 \\
\hline pHKK100 & E. faecium 228 & 55 & VanA & Handwerger et al., 1990 \\
\hline pHKK101 & E. faecium R7 & 92 & $\operatorname{Van} \mathrm{A}$ & Heaton et al., 1996 \\
\hline pBRG1 & E. faecium LS10 & 50 & $\operatorname{Van} A$ & Magi et al., 2003 \\
\hline pSL1, pSL2 & $\begin{array}{l}\text { E. faecalis KV1 } \\
\text { E. faecalis KV2 }\end{array}$ & 128 & $\begin{array}{l}\text { VanA, } \mathrm{Gm}^{\mathrm{R}}, \mathrm{Km}^{\mathrm{R}}, \mathrm{Sm}^{\mathrm{R}}, \\
\mathrm{Em}^{\mathrm{R}}\end{array}$ & Lim et al., 2006 \\
\hline pAM368 & E. faecalis 368 & 107 & VanA & Showsh et al., 2001 \\
\hline pMG2200 & E. faecalis NKH15 & 106 & VanB & Zheng et al., 2009 \\
\hline pMG2201 & E. faecalis NKH15 & 65,7 & $\mathrm{Em}^{\mathrm{R}}$ & Zheng et al., 2009 \\
\hline
\end{tabular}

bla $^{+}-\beta$-lactamase production; $\mathrm{Cm}^{\mathrm{R}}, \mathrm{Km}^{\mathrm{R}}, \mathrm{Tm}^{\mathrm{R}}, \mathrm{Gm}^{\mathrm{R}}, \mathrm{Sm}^{\mathrm{R}}, \mathrm{Em}^{\mathrm{R}}$ - resistance to chloramphenicol, kanamycin, tobramycin, gentamycin, streptomycin and erythromycin, respectively.

Dunny, 1986). Aminoglycoside and beta-lactam resistance genes were also found to be connected with pheromone plasmids. Importantly, they can be both located on the same plasmid as was the case of $70 \mathrm{~kb}$ pBEM10 encoding beta-lactamase gene, gentamycin, kanamycin and tobramycin resistance (Murray et al., 1988). This plasmid was present in multiresistant strain HH22 of E. faecalis together with two other conjugative, probably pheromone-responsive plasmids, pAM323 and pAM324.

\section{Contribution to virulence}

Enterococci are the causative agents of several serious infections encountered in hospitals, such as surgical wound infections, bloodstream and urinary tract infections (Gilmore et al., 2002) but there is still little information available about pathogenesis of these infections. Virulence traits, encoded on mobile elements can be easily transmitted within bacterial populations. Several of such traits that contribute to the severity of enterococcal infection have been characterized to varying degrees. Two of them, namely cytolysin and $\mathrm{AS}$, are carried on pheromone-responsive plasmids.

Cytolysin is a secreted virulence factor whose gene was identified on pAD1 (Borderon et al., 1982). Its discovery was one of the first indications of the possible contribution of pheromone plasmids to virulence, as it significantly enhanced perotinitis in a mouse model (Ike et al., 1984). Cytolysin displays both hemolytic and bactericidal activity. Expression of the cytolysin operon is a complex process involving the products of eight genes. Transcription starts from two divergent promoters and leads to the production of two mRNA units (Haas et al., 2002). The first transcript encodes structural genes for cytolysin subunits - $c y l L_{L}$ and $c y l L_{S}$, post-transcriptional modification and secretion functions ( $c y l M, c y l B$ and $c y l A)$ and immunity determinant (cyll). The second transcript comprises regulatory genes $-c y l R 1$ and $c y l R 2$. CylL $\mathrm{L}_{\mathrm{L}}$ and $\mathrm{CylL}_{\mathrm{S}}$ are synthesized as precursor forms and then post-translationally modified by CylM (Gilmore et al., 1994). ATP-binding cassette transporter, the product of $c y l B$ gene, mediates secretion of the subunits across the cytoplasmic membrane. CylB possesses also a cysteine protease domain and acts as signal peptidase, removing leader sequences from $\mathrm{CylL}_{\mathrm{L}}$ and $\mathrm{CylL}_{\mathrm{S}}$ precursors and generating $\mathrm{CylL}_{\mathrm{L}}$ ' and CylL $_{\mathrm{S}}$ ' (Havarstein et al., 1995). Externalized subunits undergo the last processing step - removal of six-amino-acid sequence from the $\mathrm{N}$-terminus of each subunit by subtilisin-like serine protease CylA. This leads to the generation of active toxin subunits CylL $_{\mathrm{L}}$ " and CylL ${ }_{\mathrm{S}}$ " (Segarra et al., 1991). Regulation of cytolysin expression is dependent on CylR1 and CylR2 negative regulators, as well as on the appropriate concentration of $\mathrm{CylL}_{\mathrm{S}}$ ", which acts as an autoinducer of operon functions (Haas et al., 2002). Mature cytolysin provides an effective tool for enterococci to invade different tissues and to evade the immune response of infected host (Miyazaki et al., 1993). Epidemiological data showed it to be associated with lethality in humans (Huycke et al., 1991).

The second important agent of enterococcal pathogenicity, the AS, is strictly connected with the conjugation system of pheromone-responsive plasmids. However, it displays some characteristics which can be significant in enterococcal infection mechanism. The AS is a cell wall-associated protein with the integrin-binding Arg-Gly-Asp (RGD) motifs and facilitates the adherence of enterococci to different tissues during infection (Galli et al., 1990). Some experiments showed that AS mediates binding to human polimorphonuclear leukocytes (PMN) and interfere with PMN-mediated killing (Vanek et al., 1999). 
Interestingly, the AS and cyl operon occur together on the same plasmids and likely work in concert. The AS mediates adherence to eukaryotic tissues and, as a result, the density of bacterial cells and cytolysin operon inducer $\mathrm{CylL}_{\mathrm{S}}$ " increases, which leads to the high-level expression of the toxin (Chow et al., 1993), resulting in tissue damage and invasion.

An interesting mechanism has been described in the case of cCF 10 and iCF 10 activity. It is known that the inactive state of pheromone response mechanism depends on the presence of inhibitory peptides and membrane protein PrgY. These two factors prevent the self-induction by endogenous pheromone and expression of conjugation determinants, including the AS. However, it has been reported that the pCF 10 conjugation functions can be activated in the absence of recipient cells when E. faecalis pCF10 donors are grown in human or rabbit plasma (Hirt et al., 2002). Some results indicate that albumin in human plasma interacts with iCF10 shifting the ratio of iCF10 to endogenous CCF10 facilitating the latter to self-induce the conjugation mechanism (Chandler et al., 2005). Thus, induced synthesis of AS leads to the adherence and invasion of eukaryotic tissues.

Sex pheromone plasmids seem to be an important determinant, conferring virulence traits to enterococcal clinical isolates and significant cause of antibiotic resistance spread among enterococci. Epidemiological studies show that they were more frequently found in isolates from patients with bacteremia and wound infections than from stool specimens of healthy volunteers and hospitalized patients (Coque et al., 1995).

\section{Conclusions}

Novel details of the complex genetic repertoire, successively discovered in enterococci, force us to treat them as unpredictable opportunistic pathogens with great variability potential. Our knowledge about their diverse genetic elements, among which pheromone-responsive plasmids seem to play one of the critical roles, is still just the tip of the iceberg. The group of pheromone plasmids, with their unique properties, is intriguing and mysterious, with many questions, concerning the structure, mechanism of conjugation, role and evolution of pheromone plasmids remaining open. These plasmids have developed a sophisticated and complex conjugation system restricted, as yet, only to the genus Enterococcus. Particular parts of this system often play several different roles, not only limited to conjugation itself. They seem to be responsible in some degree also for the modulation of entococcal virulence. Adding to it the participation of pheromone plasmids in dissemination of antibiotic resistance, enterococci possess sophisticated tools that help them to survive antibiotic pressure and to cause disease (Mundy et al., 2000). As a result, highly adapted hospital strains have evolved.

All interesting and complex features of pheromone-responsive plasmids constitute a perfect object to study the nature of mobile genetic elements - their biology and evolution. And finally maybe it will be possible to answer the question: "To what extent are plasmids able to evolve and influence the nature of bacterial hosts?"

\section{Acknowledgements}

We thank Dr Alicja Kuch for very helpful discussion and inspiration to create this manuscript. This work was supported by funding from the European Community (ACE contract LSHECT-2007-037410).

\section{Literature}

An F.Y. and D.B. Clewell. 2002. Identification of the cAD1 sex pheromone precursor in Enterococcus faecalis. J. Bacteriol. 184: 1880-1887.

An F.Y., M.C. Sulavik and D.B. Clewell. 1999. Identification and characterization of a determinant (eep) on the Enterococcus faecalis chromosome that is involved in production of the peptide sex pheromone cAD1. J. Bacteriol. 181: 5915-5921.

Borderon E., G. Bieth and T. Horodniceanu. 1982. Genetic and physical studies of Streptococcus faecalis hemolysin plasmids. FEMS Microbiol. Lett. 14: 51-55.

Brown M.S., J. Ye, R.B. Rawson and J.L. Goldstein. 2000. Regulated intramembrane proteolysis: a control mechanism conserved from bacteria to humans. Cell 100: 391-398.

Callen B.P., K.E. Shearwin and J.B. Egan. 2004. Transcriptional interference between convergent promoters caused by elongation over the promoter. Mol. Cell 14: 647-656.

Chandler J.R., H. Hirt and G.M. Dunny. 2005. A paracrine peptide sex pheromone also acts as an autocrine signal to induce plasmid transfer and virulence expression in vivo. Proc. Natl. Acad. Sci. USA 102: 15617-15622.

Centers for Disease Control and Prevention. National Nosocomial Infections Surveillance System (NNIS). http://www.cdc.gov/ nhsn/PDFs/dataStat/NNIS_2004.pdf

Chow J.W., L.A. Thal, M.B. Perri, J.A. Vazquez, S.M. Doanbedian, D.B. Clewell and M.J. Zervos. 1993. Plasmidassociated hemolysin and aggregation substance production contribute to virulence in experimental enterococcal endocarditis. Antimicrob. Agents Chemother. 37: 2474-2477.

Christie P.J. and G.M. Dunny. 1986. Identification of regions of the Streptococcus faecalis plasmid pCF10 that encode antibiotic resistance and pheromone response functions. Plasmid 15: 230-241.

Clewell D.B. 1993. Bacterial sex pheromone-induced plasmid transfer. Cell 73: 9-12.

Clewell D.B. 2007. Properties of Enterococcus faecalis plasmid pAD1, a member of a widely disseminated family of pheromoneresponding, conjugative, virulence elements encoding cytolysin. Plasmid. 58: 205-227.

Clewell D.B. and G.M. Dunny. 2002. Conjugation and genetic exchange in enterococci. pp. 265-300. In: Gilmore M.S. (ed.). The Enterococci: Pathogenesis, Molecular Biology, and Antibiotic Resistance. ASM Press, Washington, D.C.

Clewell D. B., F.Y. An, B.A. White and C. Gawron-Burke. 1985. Streptococcus faecalis sex pheromone (cAM373) also 
produced by Staphylococcus aureus and identification of a conjugative transposon (Tn918). J. Bacteriol. 162: 1212-1220.

Clewell D.B., F.Y. An, S.F. Flannagan, M. Antiporta and G.M. Dunny. 2000. Enterococcal sex pheromone precursors are part of signal sequences for surface lipoproteins. Mol. Microbiol. 35: 246-247.

Clewell D.B., L.T. Pontius, F.Y. An, Y. Ike, A. Suzuki and J. Nakayama. 1990. Nucleotide sequence of the sex pheromone inhibitor (iAD1) determinant of Enterococcus faecalis conjugative plasmid pAD1. Plasmid 24: 156-161.

Coque T.M., M.E. Patterson, J.M. Steckelberg and B.E. Murray. 1995. Incidence of hemolysin, gelatinase, and aggregation substance among enterococci isolated from patients with endocarditis and other infections and from feces of hospitalized and community-based persons. J. Infect. Dis. 171: 1223-1229.

Courvalin P. 2005. Genetics of glycopeptide resistance in Grampositive pathogens. Int. J. Med. Microbiol. 294: 479-486.

Courvalin P. 2006. Vancomycin resistance in Gram-positive cocci. Clin. Infect. Dis. 42: 25-34.

De Boever E.H. and D.B. Clewell. 2001. The Enterococcus faecalis pheromone-responsive plasmid pAM373 does not encode an entry exclusion function. Plasmid 45: 57-60.

Dunny G.M., B.L. Brown and D.B. Clewell. 1978. Induced cell aggregation and mating in Streptococcus faecalis: evidence for a bacterial sex pheromone. Proc. Natl. Acad. Sci. USA 75: 3470-3483.

Flannagan S.E. and D.B. Clewell. 2002. Identification and characterization of genes encoding sex pheromone cAM373 activity in Enterococcus faecalis and Staphylococcus aureus. Mol. Microbiol. 44: 803-817.

Folli C., L. Mangiarotti, S. Folloni, B. Alfieri, M. Gobbo, R. Berni and C. Rivetti. 2008. Specificity of the TraA-DNA interaction in the regulation of pPD1-encoded sex pheromone response in Enterococcus faecalis. J. Mol. Biol. 380: 932-945.

Francia M.V. and D.B. Clewell. 2002. Transfer origins in the conjugative Enterococcus faecalis plasmids pAD1 and pAM373. Identification of the pAD1 nic site, a specific relaxase and possible TraG-like protein. Mol. Microbiol. 45: 375-395.

Francia M.V., S. Fujimoto, P. Tille, K.E. Weaver and D.B. Clewell. 2004. Replication of Enterococcus faecalis pheromone responding plasmid pAD1: location of the minimal replicon and oriV site and RepA involvement in initiation of replication. J. Bacteriol. 186: 5003-5016.

Francia M.V., K.E. Weaver, P. Tille and D.B. Clewell. 2007. Characterization of an active partition system for the Enterococcus faecalis pheromone-responding plasmid pAD1. J. Bacteriol. 189: 8546-8555.

Fujimoto S. and D.B. Clewell. 1998. Regulation of the pAD1 sex pheromone response of Enterococcus faecalis by direct interaction between the CAD1 peptide mating signal and the negatively regulating, DNA-binding TraA protein. Proc. Natl. Acad. Sci. USA 95: 6430-6435.

Funnell B.E. and R.A. Slavcev. 2004. Partition systems of bacterial plasmids. pp. 81-103. In: Funnell B. E. and G. Phillips (eds). Plasmid biology. ASM Press, Washington, D.C.

Galli D., F. Lottspeich and R. Wirth. 1990. Sequence analysis of Enterococcus faecalis aggregation substance encoded by the sex pheromone plasmid pAD1. Mol. Microbiol. 4: 895-904.

Gilmore M.S., P.S. Coburn, S.R. Nallapareddy and B.E. Murray. 2002. Enterococcal virulence. pp. 301-354. In: Gilmore M.S. (ed.). The Enterococci: Pathogenesis, Molecular Biology, and Antibiotic Resistance. ASM Press, Washington, D.C.

Gilmore M.S., R.A. Segarra, M.C. Booth, C.P. Bogie, R. Hall and D.B. Clewell. 1994. Genetic structure of the Enterococcus faecalis plasmid pAD1-encoded cytolytic toxin system and its relationship to lantibiotic determinants. J. Bacteriol. 176: 7335-7344.
Grohmann E., G. Muth and M. Espinosa. 2003. Conjugative plasmid transfer in Gram-positive bacteria. Microbiol. Mol. Biol. Rev. 67: 277-301.

Haas W., B.D. Shepard and M.S. Gilmore. 2002. Two-component regulator of Enterococcus faecalis cytolysin responds to quorum-sensing autoinduction. Nature 415: 84-87.

Handwerger S., M.J. Pucci and A. Kolokathis. 1990. Vancomycin-resistance is encoded on the pheromone response plasmid in Enterococcus faecium 228. Antimicrob. Agents Chemother. 34: 358-360.

Havarstein L.S., D.B. Diep and I.F. Nes. 1995. A family of bacteriocin $\mathrm{ABC}$ transporters carry out proteolytic processing of their substrates concomitant with export. Mol. Microbiol. 16: 229-240. Heaton M.P., L.F. Discotto, M.J. Pucci and S. Handwerger. 1996. Mobilization of vancomycin resistance by transposon-mediated fusion of a VanA plasmid with an Enterococcus faecium sex pheromone-response plasmid. Gene 171: 9-17.

Hirt H., R. Wirth and A. Muscholl. 1996. Comparative analysis of 18 sex pheromone plasmids from Enterococcus faecalis: detection of a new insertion element on pPD1 and implications for the evolution of this plasmid family. Mol. Gen. Genet. 252: 640-647. Hirt H., Schlievert P.M. and G.M. Dunny. 2002. In vivo induction of virulence and antibiotic resistance transfer in Enterococcus faecalis mediated by the sex pheromone-sensing system of pCF10. Infect. Immun. 70: 716-723.

Huycke M.M., C.A. Spiegeland and M.S. Gilmore. 1991. Bacteremia caused by hemolytic, high-level gentamicin resistant Enterococcus faecalis. Antimicrob. Agents. Chemother. 35: 1626-1634.

Ike Y., H. Hashimoto and D.B. Clewell. 1984. Hemolysin of Streptococcus faecalis supspecies zymogenes contributes to virulence in mice. Infect. Immun. 45: 528-530.

Kozlowicz B.K., M. Dworkin and G.M. Dunny. 2006. Pheromone-inducible conjugation in Enterococcus faecalis: a model for the evolution of biological complexity? Int. J. Med. Microbiol. 296:141-7.

Lim S.K., K.Tanimoto, H. Tomita and Y. Ike. 2006. Pheromoneresponsive conjugative vancomycin resistance plasmids in Enterococcus faecalis isolates from humans and chicken feces. Appl. Environ. Microbiol. 72: 6544-6553.

Magi G., R. Capretti, C. Paoletti, M. Pietrella, L. Ferrante, F. Biavasco, P.E. Varaldo and B. Facinelli. 2003. Presence of a vanA-carrying pheromone response plasmid (pBRG1) in a clinical isolate of Enterococcus faecium. Antimicrob. Agents Chemother. 47: 1571-1576.

Miyazaki S., A. Ohno, I. Kobayashi, T. Uji, K. Yamaguchi and S. Goto. 1993. Cytotoxic effect of hemolytic culture supernatant from Enterococcus faecalis on mouse polymorphonuclear neutrophils and macrophages. Microbiol. Immunol. 37: 265-270.

Mori M., A. Isogai, Y. Sakagami, M. Fujino, C. Kitada, D.B. Clewell and A. Suzuki. 1986. Isolation and structure of Streptococcus faecalis sex pheromone inhibitor, iAD1, that is excreted by donor strains harboring plasmid pAD1. Agric. Biol. Chem. 50: 539-541.

Mundy L.M., D.F. Sahm and M. Gilmore. 2000. Relationships between enterococcal virulence and antimicrobial resistance. Clin. Microbiol. Rev. 13: 513-522.

Murray B.E. 1998. Diversity among multidrug-resistant enterococci. Emerg. Infect. Dis. 4: 37-47.

Murray B.E., F.Y. An and D.B. Clewell. 1988. Plasmids and pheromone response of the $\beta$-lactamase producer Streptococcus (Enterococcus) faecalis HH22. Antimicrob. Agents Chemother. 32: 547-551.

Muscholl-Silberhorn A. 1999. Cloning and functional analysis of Asa373, a novel adhesin unrelated to the other sex pheromone plasmid-encoded aggregation substances of Enterococcus faecalis. Mol. Microbiol. 34: 620-630. 
Ozawa Y., E.H. De Boever and D.B. Clewell. 2005. Enterococcus faecalis sex pheromone plasmid pAM373: Analyses of TraA and evidence for its interaction with RpoB. Plasmid 54: 57-69.

Pontius L.T. and D.B. Clewell. 1992. Conjugative transfer of Enterococcus faecalis plasmid pAD1: nucleotide sequence and transcriptional fusion analysis of a region involved in positive regulation. J. Bacteriol. 174: 3152-3160.

Segarra R.A., M.C. Booth, D.A. Morales, M.M. Huycke and M.S. Gilmore. 1991. Molecular characterization of the Enterococcus faecalis cytolysin activator. Infect Immun 59: 1239-1246.

Showsh S.A., H. De Boever and D.B. Clewell. 2001. Vancomycin resistance plasmid in Enterococcus faecalis that encodes sensitivity to a sex pheromone also produced by Staphylococcus aureus. Antimicrob. Agents Chemother. 45: 2177-2178.

Tomita H. and D.B. Clewell. 2000. A pAD1-encoded small RNA molecule, mD, negatively regulates Enterococcus faecalis pheromone response by enhancing transcription termination. J. Bacteriol. 182: 1062-1073.

Vanek N.N., S.I. Simon, K. Jacques-Palaz, M.M. Mariscalco, G.M. Dunny and R.M. Rakita. 1999. Enterococcus faecalis aggregation substance promotes opsonin-independent binding to human neutrophils via a complement receptor type 3-mediated mechanism. FEMS Immunol. Med. Microbiol. 26: 49-60.

Weaver K.E., D.M. Weaver, C.L. Wells, C.M. Waters, M.E. Gardner and E.A. Ehli. 2003. Enterococcus faecalis plasmid pAD1-encoded Fst toxin affects membrane permeability and alters cellular responses to lantibiotics. J. Bacteriol. 185 2169-2177.
Weaver K.E., E.A. Ehli, J.S. Nelson and S. Patel. 2004. Antisense RNA regulation by stable complex formation in the Enterococcus faecalis plasmid pAD1 par addiction system. $J$. Bacteriol. 186: 6400-6408.

Weaver K.E., L.B. Rice and G. Churchward. 2002. Plasmids and transposons. pp. 219-263. In: Gilmore M. S. (ed.). The Enterococci: Pathogenesis, Molecular Biology, and Antibiotic Resistance. ASM Press, Washington, D.C.

Weaver K. E., S.M. Kwong, N. Firth and M.V. Francia. 2009. The RepA N replicons of Gram-positive bacteria: A family of broadly distributed but narrow host range plasmids. Plasmid 61: 94-109.

Wirth R. 1994. The sex pheromone system of Enterococcus faecalis. More than just a plasmid-collection mechanism? Eur. J. Biochem. 222: 235-246.

Yagi Y., R.E. Kessler, J.H. Shaw, D.E. Lopatin, F. An and D.B. Clewell. 1983. Plasmid content of Streptococcus faecalis strain 39-5 and identification of a pheromone (cPD1)-induced surface antigen. J. Gen. Microbiol. 129: 1207-15.

Zheng B., H. Tomita, T. Inoue, and Y. Ike. 2009. Isolation of VanB-type Enterococcus faecalis strains from nosocomial infections: first report of the isolation and identification of the pheromone-responsive plasmids pMG2200, encoding VanB-type vancomycin resistance and a Bac41-type bacteriocin, and pMG2201, encoding erythromycin resistance and cytolysin (Hly/Bac). Antimicrob. Agents Chemother. 53: 735-47.

Zielenkiewicz U. and P. Ceglowski. 2001. Mechanisms of plasmid stable maintenance with special focus on plasmid addiction systems. Acta Biochim. Pol. 48: 1003-23. 